\title{
Maximum entropy production as a necessary admissibility condition for the fluid Navier-Stokes and Euler equations
}

\author{
James Glimm ${ }^{1,2}$ • Daniel Lazarev ${ }^{1,3,4} \cdot$ Gui-Qiang G. Chen ${ }^{5}$
}

Received: 15 June 2020 / Accepted: 21 November 2020 / Published online: 5 December 2020

(c) Springer Nature Switzerland AG 2020

\begin{abstract}
In a particle physics dynamics, we assume a uniform distribution as the physical measure and a measure-theoretic definition of entropy on the velocity configuration space. This distribution is labeled as the physical solution in the remainder of the article. The dynamics are governed by an assumption of a Lagrangian formulation, with the velocity time derivatives as the momenta conjugate to the velocity configurations. From these definitions and assumptions, we show mathematically that a maximum entropy production principle selects the physical measure from among alternate solutions of the Navier-Stokes and Euler equations, but its transformation to an Eulerian frame is not established here, a topic that will be considered separately.
\end{abstract}

Keywords Navier-Stokes · Euler equations · Maximum entropy production · Admissibility conditions · Nonuniqueness . Jensen's inequality

Mathematical Subject Classification $46 \mathrm{~A} 13 \cdot 37 \mathrm{~A} 50$

\section{Introduction}

\subsection{Background}

Turbulence is perhaps the foremost problem of classical (nonquantum) physics. The main result of this paper is a proof of a maximum rate of entropy production as a necessary admissibility principle for physical solutions of fluid turbulence. For a mathematical analysis, as we present here, we define the physical measure on the fluid velocity configuration space as proportional to the uniform distribution.

Our result is a necessary condition for the admissibility of solutions of the Navier-Stokes and Euler equations of fluid dynamics. The examples of nonunique solutions of the Navier-Stokes equations [5] and the Euler equations
$[6,20,25,35]$ show the need for such an admissibility principle.

For fluid mixing, an entropy of mixing, similar in form to the entropy considered in this paper, but defined based on concentration gradients, is subject to the same physical laws of entropy maximization. The total entropy of a turbulent fluid can be decomposed into a sum of a configurational or thermal entropy related to fluid density fluctuations, an entropy of mixing related to (thermal) concentration gradients, and a kinetic energy entropy related to fluid velocity fluctuations. For a constant density fluid, that is, classical turbulence, as considered here, the entropy is exclusively kinetic, and related to the fluid velocities.

Daniel Lazarev, dlazarev@broadinstitute.org; James Glimm, glimm@ams.sunysb.edu; Gui-Qiang G. Chen, chengq@maths.ox.ac.uk | ${ }^{1}$ Stony Brook University, Stony Brook, NY 11794, USA. ${ }^{2}$ GlimmAnalytics LLC, New York, USA. ${ }^{3}$ Stanley Center for Psychiatric Research, Broad Institute of MIT and Harvard, Cambridge, MA 02142, USA. ${ }^{4}$ Analytic and Translational Genetics Unit, Massachusetts General Hospital, Boston, MA 02114, USA. ${ }^{5}$ University of Oxford, Oxford OX2 6GG, UK. 
Theorem 1 The physical measure maximizes the entropy production rate in comparison with alternate measures on the velocity configuration space.

The distinction between Ziegler's principle of maximum entropy production $[39,40]$ and Prigogine's principle of minimum entropy production [33] lies in the distinction between open and closed systems. In an open system, the entropy, which must increase for any irreversible processes, can escape to the external world and not be present in the model. For a closed system, there is no such escape. Thus, we view Ziegler's principle as applicable to closed systems and Prigogine's principle as applicable to open ones. To illustrate this distinction, consider the irreversible experiment of dropping a stone from the tower of Pisa. The entropy, which must increase due to the irreversibility of the experiment, is not found in the stone. Considered in isolation, the stone is an open system and its entropy increase is minimized (zero) according to Prigogine's principle. The entropy does increase within the air disturbed by the falling stone. This disturbance accounts for the aerodynamic (turbulent) drag on the falling stone and, if included in the model, the system has a maximum rate of increase, according to Ziegler's principle. Therefore, we see the Prigogine-Ziegler distinction not as a controversy regarding laws of physics but as alternate modeling strategies in the construction of a physical model.

All models involve approximations or idealizations. The selection of a model is a judgment on the part of the modeler and is not uniquely determined by the problem. For this reason, the two opposite principles coexist. In the case of observational or experimental turbulence, the dominant dissipation and entropy production occur in boundary layers. Rather than modeling boundary layer entropy production explicitly, carefully controlled measurements of turbulence are generally located in regions of space far from such boundaries. This convention explains the fact that turbulence measurements generally support Ziegler's principle and not Prigogine's principle. Thus, the main result of the program proposed here, in support of Ziegler's principle, is that classical fluid turbulence is a closed system.

The role of stirring forces brings this distinction into sharper focus. Stirring forces are governed by ideas of Prigogine, while the non-stirred, or what we call Never Stirred (NevS) set of initial conditions and of Navier-Stokes solutions (which have no memory of past stirring) are governed by Ziegler's rule, according to which the physical solution selects the entropy maximizing solution from among the nonunique solutions of the Navier-Stokes equations. This set of initial conditions is time invariant. Put differently, the physical solution belongs to a NevS subspace, velocity configurations stirred neither in the past nor in the present nor in the future. This is the subspace of Navier-Stokes initial conditions to which the maximum entropy condition applies. From this subspace, the general solution, with stirring in the past, present or future, is readily constructed by coordinate transformations, thereby recovering a general solution from a NevS one.

Maximum entropy production rates and maximum energy dissipation rates are very general scientific principles, and their importance extends well beyond the single fluid turbulence considered here. Often interfaces or near interfaces defined by narrow diffusion layers arise in the study of fluid mixtures. Efforts to improve the modeling of atmospheric turbulent boundary layers can be based on the maximum dissipation rate idea emphasized here. Likewise, energy transfer within the Inertial Confinement Fusion (ICF) capsule is subject to plasma instabilities, likely of a turbulent origin, and the resulting boundary layer enhanced ablation at the ICF capsule surface that has been observed is likely a turbulent boundary layer interaction, deleterious to the ICF yield. Experimental studies of acceleration driven fluid mixing are typically performed either with a sharp interface (immiscible fluids) or with a nearly immiscible mixture (high Batchelor number). Thus, we see multiple reasons to extend the present analysis to mixtures and in doing this, to consider the role interfaces as defining the nature of the mixtures.

\subsection{Prior studies}

The maximum entropy production principle (MEPP), as a principle of physics, has a long history, of which we cite $[12,13,28,29]$. This principle has been used in two key ways:

(i) to derive an evolution system of equations;

(ii) to select the physically relevant solution of a given evolution system.

\subsubsection{Particle physics}

MEPP has been used to derive laws such as Kirchhoff's circuit laws [41] and Fourier's law [27]. Jordan, Kinderlehrer, and Otto showed that the heat equation is the gradient flow, or steepest descent, of a functional equal to the negative of the Boltzmann-Gibbs entropy $[22,23]$. A proof of entropy maximization for lattice gases is given by Lanford [24]. 


\subsubsection{Navier-Stokes and Euler equations: prior results}

As a selection principle, MEPP has been used in a variety of applications, from the study of enzyme kinetics [14] to the spatial organization of vegetation in river basins [21]. In the physical analysis of fluid turbulence, MEPP has been successfully used to select the physical solution for the evolution of the global climate system [31, 32]. In regard to the Euler equations, successful results have been obtained for the two-dimensional case by Chavanis et al. [8], who maximized the mixing entropy to obtain the equilibrium distribution, given standard energy and momentum constraints, and then used MEPP to derive the equations governing the evolution of the configuration space probability densities. Boucher et al. [2] derived maximum entropy principles for continuum models of two-dimensional turbulence by using the theory of large deviations. Thalabard et al. [37] used entropy maximization to select solutions for the three-dimensional axisymmetric Euler equations in a Taylor-Couette geometry. Axisymmetric flow in 3D is also two-dimensional.

Recently, an exact solution to the compressible 3D Navier-Stokes equations was obtained by using a relation between the pressure and the divergence of the velocity, which was in turn derived from the positive-definiteness of the entropy production rate, a weaker form of MEPP $[9,10]$.

\section{Properties of the physical measure}

The physical measure is defined as proportional to the Lebesgue measure in a framework of particle physics. We identify two basic properties or restrictions on the physical measure. It must

(i) be restricted to the entropy of indistinguishable particles only;

(ii) be restricted to the surface of constant energy.

Both of these conditions are then imposed on the alternate measures used for comparison to the physical measure.

\subsection{The Navier-Stokes equations}

Definition 1 Let $V$ be a finite cube in $\mathbb{R}^{3}$, and $[0, T]$ a finite time interval. Let $\mathcal{H}$ be the $L_{2}$ space of divergence-free velocity fields defined over $V$ with periodic boundary conditions. The distinguishable particle configuration space $\mathcal{V}^{d}(V \times[0, T])$ is the space $\mathcal{M}([0, T] ; \mathcal{H})$ of Radon measures of $t \in[0, T]$ with values in $\mathcal{H}$.
The physical measure and all comparison measures are realized as linear functionals defined on the configuration space $\mathcal{V}^{d}$. In view of the self-duality for any Hilbert space $\mathcal{H}$, we identify the dual $\mathcal{M}\left([0, T] ; \mathcal{H}^{*}\right)$ as the space $\mathcal{C}([0, T] ; \mathcal{H})$ of continuous functions on $[0, T]$ with values in $\mathcal{H}$. We use the weak topology on $\mathcal{M}$ to define the open sets. The Borel sets in $\mathcal{M}$ are members of the $\sigma$-ring generated by the open sets.

Definition 2 The physical measure on the configuration space $\mathcal{V}$ is proportional to the Lebesgue measure.

The physical measure is invariant under translations by velocities $u \in \mathcal{V}$ and by two independent angular rotations. The first modification of the physical measure is the specification of the Navier-Stokes (NS) equations in the Lagrangian frame which it solves. With a viscosity $v(x, t)$ given, the Lagrangian Navier-Stokes equations at fixed time $t$ are

$\frac{D}{D t} u(x, t)=\nabla \cdot(v(x, t) \nabla u(x, t))+\nabla p$,

where $D / D t$ is the material derivative, and $u(x, t) \in \mathcal{H}$.

In the study of the NS equations, it is documented in, e.g., [4], that the pressure can be eliminated by projection onto the divergence-free subspace. This approach does not eliminate the pressure from the equations, nor does it change the meaning of the equations themselves, but rather it "hides" the pressure. Projecting onto the divergence-free subspace of $\mathcal{H}$, we rewrite (1) as

$\partial_{t} u(x, t)=\mathbf{P}[-(u(x, t) \cdot \nabla) u(x, t)+\nabla \cdot(v(x, t) \nabla u(x, t))]$,

where $\mathbf{P}$ is the operator projecting the inputted vector field onto the divergence-free subspace with the appropriate boundary conditions.

Particles in the context of the NS equations carry distinct and different possible meanings. We are considering particles as descriptive of the thermodynamic degrees of freedom for energy and entropy, in the manner used by [24]. These particles are points in the NS fixed time configuration space, which is to say that they are NS velocity fields, and in our case, periodic velocity fields in a finite volume $V$ with periodic boundary conditions and which are divergence free.

Quite unrelated are the particles inserted in a NS flow and advected with the flow [16]. Such particles are useful for the study of transport phenomena and mixing, among other applications of this methodology. In this context, omission of the pressure term leads to the Burger's equations, which are essentially the pressureless NS equations.

Comparing these two sets of ideas, we see that the Burger's equations describe a physics that is fundamentally 
modified from the NS equations, while the use of a divergence-free subspace to hide the pressure does not alter in any manner the physics of the NS equations, which are equations for the diffusion of momentum in fluid flow. We see that the particles are Lagrangian trajectories within a NS flow in one case, and are incompressible velocity fields in the other case.

As $v$ enters in (1) or (2) multiplying second velocity derivatives, it is natural to require $u(x, t) \in \mathcal{H}_{+2}$, the Sobolev space of functions with two $L_{2}$ spatial derivatives. Since $u$ lies in the dual space of functions continuous in $t$, we suppose that $u(x, t) \in C\left([0, T], \mathcal{H}_{+2}\right)$ of continuous functions of time with values in $\mathcal{H}_{+2}$. The regularity condition is weakened to $u \in \mathcal{H}$ through the use of cylinder sets (defined below) with smooth basis elements, and projective limits.

We impose the solution restriction (1) or (2) of the Navier-Stokes equations on the comparison measures to which the physical measure is compared. The validity of these equations for the physical solution will be addressed separately.

\subsection{Transformation group invariance properties}

The subspace of the configuration space $\mathcal{V}$ defined by the Navier-Stokes (or Euler) Eqs. (1) or (2) is invariant by elements of the semidirect product of the group of translations by a velocity $u(x, t)$ in the subspace defined by the Navier-Stokes (or Euler) Eqs. (1) or (2) , and the orthogonal group of transformations on the vector indices of the velocity.

The consequence of these symmetries, namely Noether's theorem, according to which the velocity field $u(x, t)$ in the Navier-Stokes physical solution subspace of configuration space $\mathcal{H}$ conserves linear momentum and two independent angular momenta as a thermodynamic identity [30], will be established separately. Conservation of linear momentum is equivalent to the Navier-Stokes equations, i.e., (1) or (2).

\subsection{Indistinguishability}

We remove the entropy distinction between labels for individual particles. This goal is achieved through the definitions of distinguishable and indistinguishable configuration spaces and the linear functionals on them. To identify individual particles (distinguished or not) within the particle physics formalism, we introduce cylinder sets defined by finite dimensional subspaces $\mathcal{H}_{n}$ of $\mathcal{H}$. To illustrate the ideas, we work out the details explicitly for $n=2$ particles at a fixed time $t \in[0, T]$. The two particle configuration space $\mathcal{H}_{2}$ is the set of 2-tuples, $\left(u_{1}\left(x_{1}\right), u_{2}\left(x_{2}\right)\right) \in \mathcal{H} \times \mathcal{H}$. If the particles are distinguishable, then states $\left(u_{1}\left(x_{1}\right), u_{2}\left(x_{2}\right)\right)$ and $\left(u_{2}\left(x_{2}\right), u_{1}\left(x_{1}\right)\right)$ are distinct. If they are indistinguishable, we consider the symmetry group $\mathcal{S}_{2}$ on two objects, and regard the orbits under $\mathcal{S}_{2}$ acting on $\mathcal{H}_{2}$ as the elementary points in the indistinguishable 2 particle configuration space. Given a measure $\mu_{2}^{d}$ on the distinguishable 2 particle configuration space, we construct an indistinguishable measure $\mu_{2}$ on the indistinguishable configuration space by the formula

$\mu_{2}=\frac{1}{2 !} \mu_{2}^{d}=\frac{1}{\left|\mathcal{S}_{2}\right|} \sum_{s_{2} \in \mathcal{S}_{2}} \mu_{2}^{d} s_{2}\left(u_{1}\left(x_{1}\right), u_{2}\left(x_{2}\right)\right)$

where $\left|\mathcal{S}_{2}\right|=2$ ! is the number of elements in $\mathcal{S}_{2}$. Thus, the division by 2 ! is identical to taking the average of the distinguishable measure of the members of the orbit, averaged over the orbit. These definitions and calculations generalize to $\mathcal{H}_{n}$ and $\mathcal{S}_{n}$.

We assume that a distinguished basis has been chosen for $\mathcal{H}$, and let $\mathcal{H}_{n}$ be the span of the first $n$ basis elements. Using this (self-dual) space, we define the cylinder sets which allow us to define distinguishability and indistinguishability.

We consider a fixed time $t \in[0, T]$ and define the fixed time cylinder set $\left(\delta_{t}, \mathcal{H}_{n}\right) \subset \mathcal{M}([0, T] ; \mathcal{H})$ of configuration space and its dual $\left(t, \mathcal{H}_{n}\right) \subset(t, \mathcal{H})$ of functionals on the configuration space. Any Borel set $A_{n} \subset \mathcal{H}_{n}$ similarly defines a Borel cylinder set at fixed time.

With this preparation, we define the subspaces of totally distinguishable and totally indistinguishable configuration spaces and the linear functionals on these configuration space. Relative to the basis for $\mathcal{H}$, consider the symmetry group $\mathcal{S}_{n}$ on $n$ objects, acting on the first $n$ coefficients of the velocity when expanded in this chosen basis. An element of $\mathcal{H}_{n}$ is totally distinguishable, while its orbit under $\mathcal{S}_{n}$ has $n$ ! elements and is totally indistinguishable. These definitions also apply to functionals on the configuration space.

Given a totally distinguishable linear functional $\mu^{d}$ on the $n$ particle fixed time configuration space, we associate to it a new indistinguishable functional $\mu$ on the indistinguishable particle configuration space, as the average of $\mu^{d}$ over the symmetry group orbit. This average is the original measure of the orbit (the sum over the $n$ ! orbit elements) divided by $n !$. In the case of subspace inclusion $\mathcal{H}_{m} \subset \mathcal{H}_{n}$, consistency requirements involving the $n$ ! and $m$ ! are readily verified. These same consistency relations are the formal definition of projective limits for groups, so that an infinite dimensional symmetry group is defined on the configuration space and linear functionals on the configuration space. The consistent linear functionals $\mu$ satisfy the criteria of a projective limit and define a finitely additive fixed time linear functional on the configuration space. 
We require the basis elements in $\mathcal{H}_{n}$ to be smooth functions of $x \in V$, allowing spatial derivatives to be evaluated within cylinder sets.

We use the notion of projective limit to pass from cylinder sets to an infinite space limit [3, 34].

Definition 3 Consider a projective system $\left(X_{n}, g_{m n}\right)$ defined by an object $X_{n}$ and maps $g_{m n}: X_{n} \rightarrow X_{m}$ satisfying the consistency condition:

$g_{l m} \circ g_{m n}=g_{l n} \quad$ for $\quad I \leq m \leq n$.

Then the projective limit is $\left(X, g_{n}\right)$, where $X=\lim X_{n}$ is an object and $g_{n}$ is a map $g_{n}: X \rightarrow X_{n}$ satisfying:

$g_{m}=g_{m n} \circ g_{n}$

$\left(X, g_{n}\right)$ is universal if, for any other pair $\left(Y, f_{n}\right)$ with $f_{n}: Y \rightarrow X_{n}$ satisfying [2.3], there is a unique morphism $k: Y \rightarrow X$ such that $f_{n}=g_{n} \circ$.

Proposition 1 The system $X$ consists of the following structures:

(i) $\left(t, \mathcal{H}_{n}\right), t \in[0, T]$,

(ii) $A_{\text {Borel }} \subset \mathcal{H}_{n^{\prime}}$

(iii) $\mathcal{S}_{n}$

(iv) indistinguishable particle configuration space and linear functionals $\mu_{n}$.

The consistency conditions of Definition 3 are satisfied, as are the universality conditions of the projective limit thus defined.

Proof We consider the morphisms for each of the cases individually:

(i) $g_{m n}^{-1}: \mathcal{H}_{m} \rightarrow \mathcal{H}_{n}$ is the inclusion map of a smaller Hilbert space to a larger one.

(ii) $g_{m n}^{-1}$ is an inclusion map.

(iii) $g_{m n}^{-1}: \mathcal{S}_{m} \rightarrow \mathcal{S}_{n}$ is the inclusion map from a smaller symmetry group to a larger one. It is readily verified that (i)-(iii) satisfy the necessary consistency conditions.

(iv) Let $\mathcal{V}_{1}, \mathcal{V}_{m}$, and $\mathcal{V}_{n}$ be the indistinguishable cylinder subsets of $\mathcal{V}$ such that $\mathcal{V}_{l} \subset \mathcal{V}_{m} \subset \mathcal{V}_{n}$, and let $\mu_{l}, \mu_{m}$, and $\mu_{n}$ be the measures defined on them, respectively.

Without loss of generality, we let their dimensions (denoted by their respective subscripts) satisfy $I<m<n$. Define a restriction map $g_{m n}: \mathcal{V}_{n} \rightarrow \mathcal{V}_{m}$ given by $g_{m n}\left(A_{n}\right)=\frac{m !}{n !} A_{m}$, where $A_{l} \subseteq \mathcal{V}_{1}, A_{m} \subseteq \mathcal{V}_{m^{\prime}} A_{n} \subseteq \mathcal{V}_{n^{\prime}}$ and $\mu_{l}^{d}\left(A_{l}\right)=\mu_{m}^{d}\left(A_{m}^{n !}\right)=\mu_{n}^{d}\left(A_{n}\right)$. Then

$$
\begin{aligned}
g_{l m} \circ g_{m n}\left(A_{n}\right) & =g_{l m}\left(\frac{m !}{n !} A_{m}\right) \\
& =\frac{l !}{m !} \frac{m !}{n !} A_{l} \\
& =\frac{1 !}{n !} A_{l} \\
& =g_{l n}\left(A_{n}\right) .
\end{aligned}
$$

The linear functionals on the indistinguishable particle configuration space equal those on the distinguishable particle configuration space, after division by a factor of $n$ !. Given the restriction map $g_{m n}$, we verify that these indistinguishable particle linear functionals are consistent:

$$
\begin{aligned}
\mu_{m} \circ g_{m n}\left(A_{n}\right) & =\mu_{m}\left(\frac{m !}{n !} A_{m}\right) \\
& =\frac{1}{m !} \frac{m !}{n !} \mu_{m}^{d}\left(A_{m}\right) \\
& =\frac{1}{n !} \mu_{n}^{d}\left(A_{n}\right) \\
& =\mu_{n}\left(A_{n}\right) .
\end{aligned}
$$

We next prove the universality of the projective limit. Consider the projective limits $P_{1}$ and $P_{2}$, each defined by an ordered basis set such that $P_{1} \subset P_{2}$ if every basis element of $P_{1}$ occurs within the $P_{2}$ basis list.

We verify the conditions for $g_{n m}^{-1}$ rather than those for $g_{m n}$. These are simply subset relations and are trivially consistent, other than the $n$ ! analysis for indistinguishable objects, which are verified as before.

If $P_{1} \subset P_{2}$ and $P_{2} \subset P_{1}$, then the two basis sets are identical up to reordering. In this case, we have the identity of the projective limits, so that we write $P_{1}=P_{2}$.

Given general $P_{1}$ and $P_{2}$, neither subsets of the other, we consider the projective limit $\mathcal{P}$ which chooses basis elements alternately from the $P_{1}$ and $P_{2}$ basis sets. Then $P_{1} \subset \mathcal{P}$ and $P_{2} \subset \mathcal{P}$, and each is a subset of the projective limit $\mathcal{P}$.

The universal limit $\mathcal{P}$ is the union over all possible projective limits $P_{i}$, and it is universal in that any projective limit is a subset of $\mathcal{P}$.

Projective limits are used to define the physical (Lebesgue) measure on the configuration space of distinguishable velocity values, the symmetry group of velocity interchanges, the configuration space of indistinguishable velocity values (which is the quotient space of equivalence classes under this symmetry group), and the physical (Lebesgue) measure on the indistinguishable velocity configuration space.

Limits of consistent measures to a finitely additive limit are known as a special case of this formalism. The formalism serves mainly to clarify the conceptual issues relating 
to the limit over cylinder sets. We proceed at the level of cylinder sets, where most of the analysis of the paper occurs.

Definition 4 The physical measure $\mu_{n}^{d, *}$ on an $n$-dimensional cylinder subset of the distinguishable configuration space is defined by the Lebesgue measure on that cylinder subset.

The physical measure $\mu^{d, *}$ on the distinguishable particle configuration space is the finitely additive projective limit of the projective system defined by the cylinder subsets and the physical cylinder set measures on them.

The factor $n$ ! accounts for the fact that the interchange of velocities $u_{i} \leftrightarrow u_{j}$ defines an identical point in the configuration space of indistinguishable particles.

Definition 5 The indistinguishable particle configuration space of order $n, \mathcal{V}_{n}$, is the set of equivalence classes of the cylinder set configuration space of dimension $n$ under the particle interchange symmetry. The indistinguishable physical measure $\mu_{n}^{*}=\mu_{n}^{d, *} / n$ ! is the Lebesgue measure divided by $n$ !.

The physical measure $\mu^{*}$ on the indistinguishable particle configuration space is the finitely additive projective limit of the projective system defined by the cylinder subsets and the physical cylinder set measures on them.

\subsection{The energy surface}

Thermodynamically, entropy is a function of two thermodynamic variables, density $\rho$ and energy $e$. These can be specified in a variety of ways, depending on the thermodynamic ensemble assumed. In the microcanonical ensemble, both $\rho$ and $e$ have prescribed values. We set the density $\rho=1$, so that the energy $e$ is given by $e(u)(x, t)=\left(u^{2} / 2\right)(x, t)=u(x, t)^{2} / 2$. We specify $e(u)(t)=\|e(u)(\cdot, t)\|_{L_{1}(v)}$ as defining the fixed time energy surface to be imposed as a constraint on the configuration space.

Definition 6 A (fixed time) candidate measure $\mu_{n}$ on the indistinguishable particle configuration space is a consistent family of indistinguishable particle Radon measures defined on the cylinder set configuration space $\mathcal{V}_{n}$ such that each cylinder set measure is absolutely continuous with respect to the (fixed time) indistinguishable particle Lebesgue measure. The measure is assumed to be restricted to the energy surface and to be invariant under the orthogonal group and translations, both acting on the velocities.

The physical measure at fixed time is a candidate measure.

Proposition 2 Fix a value of $t \in[0, T]$, and let $\mu_{n}$ be a candidate measure on the distinguishable particle cylinder set configuration space. Let $\mathcal{V}_{n}(e, V)$ denote the energy surface of the cylinder subset of the configuration space with $r=r(e)$ as its radius, and let $f=f(u)$ be a continuous functional of the velocities $u$ in $\mathcal{V}_{n}(e, V)$. The restriction of the Radon measure $\mu_{n}$ to $e(u)(t)$ is defined as the measure $\mu_{n, e}$ on $\mathcal{V}_{n}(e, V)$ given by

$\left.\int f\right|_{e(u)=e} \mathrm{~d} \mu_{n, e}=\left.\left(\frac{\mathrm{d}}{\mathrm{d} r} \int f \mathrm{~d} \mu_{n}\right)\right|_{r=r(e)}$.

In other words, on the left-hand side, $f$ is restricted to the energy surface and, on the right-hand side, the integral of $f$ is evaluated at the same value of $e$.

Proof We change variables to a radial variable and $n-1$ angular variables. The Jacobian for this transformation is smooth away for $r=0$. In these coordinates, the evaluation of the integral is elementary.

\section{Measure theoretic entropy}

The thermodynamic entropy is defined by Boltzmann and Gibbs as

$S_{B G S}\left(\sigma, \mathcal{V}_{n}(e, V)\right)=-\int_{\mathcal{V}_{n}(e, V)} \sigma \log (\sigma) \mathrm{d}^{3} v$,

where $\sigma$ is a probability density function $[15,17,38]$. The information-theoretic entropy defined by Shannon for discrete random variables is extended to continuous random variables by a similar formula [11]. A generalization of these definitions is the Baron-Jauch entropy $[1,36,38]$.

Definition 7 The (Baron-Jauch) entropy defined by a probability measure $\mu_{e}$ on a measure space $\mathfrak{B}$ relative to a measure $v_{e}$ on $\mathfrak{V}$ (where $\mu_{e}$ is absolutely continuous with respect to $v_{e}$ ) is

$S\left(\mu_{e}, \mathfrak{Z}\right)=-\int_{\mathfrak{B}}\left(\frac{\mathrm{d} \mu_{e}}{\mathrm{~d} v_{e}}\right) \log \left(\frac{\mathrm{d} \mu_{e}}{\mathrm{~d} v_{e}}\right) \mathrm{d} v_{e}$.

In what follows, we take $\mathfrak{B}=\mathcal{V}_{n}(e, V)$, the cylinder subset of the configuration space $\mathcal{V}$ restricted to the energy 
surface, and use the corresponding cylinder set measure. This entropy depends on the reference measure $v_{e}$. It was shown [15] that the Baron-Jauch entropy (5) agrees with the thermodynamic entropy (4) when the reference measure is the Lebesgue measure. Thus, we let $v_{e}$ be the indistinguishable particle Lebesgue measure restricted to the energy surface.

Remark 1 The formula (5) can be extended to define the entropy for a finite but not necessarily unitary measure restricted to the energy surface. By defining the probability measure in (5) as

$\mu_{e}(X)=\frac{\eta_{e}(X)}{\eta_{e}\left(\mathcal{V}_{n}(e, V)\right)}$,

with $\eta_{e}(X)$ being a finite measure, we obtain

$$
\begin{aligned}
& S\left(\eta_{e}, \mathcal{V}_{n}(e, V)\right)=\log \eta_{e}\left(\mathcal{V}_{n}(e, V)\right) \\
& \quad-\frac{1}{\eta_{e}\left(\mathcal{V}_{n}(e, V)\right)} \int_{\mathcal{V}_{n}(e, V)}\left(\frac{\mathrm{d} \eta_{e}}{\mathrm{~d} v_{e}}\right) \log \left(\frac{\mathrm{d} \eta_{e}}{\mathrm{~d} v_{e}}\right) \mathrm{d} v_{e} .
\end{aligned}
$$

All our results below hold for finite measures by using (6) instead of (5) in the proofs. However, proceeding with (5) and probability measures allows for cleaner, less congested proofs. Using (6) in the proofs, one can readily verify that all our results for the physical measure are obtained for any measure proportional to the Lebesgue measure (i.e., for the Lebesgue measure with a constant prefactor).

\section{Maximum entropy and admissibility}

A key step in the proof of our main theorem, Theorem 1, is a reformulation of the entropy defined by the physical measure.

Proposition 3 The entropy defined by the physical measure is given by

$S\left(\mu_{n, e^{\prime}}^{*} \mathcal{V}_{n}(e, V)\right)=\log \left|\mathcal{V}_{n}(e, V)\right|$,

where $|\cdot|=v_{e}(\cdot)$ denotes the indistinguishable particle Lebesgue measure restricted to the energy surface.

Proof We write $\mu_{n, e}^{*}(X)=|X| /\left|\mathcal{V}_{n}(e, V)\right|$. Then its RadonNikodym derivative is

$\frac{\mathrm{d} \mu_{n, e}^{*}}{\mathrm{~d} v_{e}}=\frac{1}{\left|\mathcal{V}_{n}(e, V)\right|}$

Starting with (5) with $\mu_{n, e}=\mu_{n, e^{\prime}}^{*}$ we have

$$
\begin{aligned}
& S\left(\mu_{n, e^{\prime}}^{*} \mathcal{V}_{n}(e, V)\right) \\
& =-\int_{\mathcal{V}_{n}(e, V)}\left(\frac{\mathrm{d} \mu_{n, e}^{*}}{\mathrm{~d} v_{e}}\right) \log \left(\frac{\mathrm{d} \mu_{n, e}^{*}}{\mathrm{~d} v_{e}}\right) \mathrm{d} v_{e} \\
& =-\int_{\mathcal{V}_{n}(e, V)}\left(\frac{1}{\left|\mathcal{V}_{n}(e, V)\right|}\right) \log \left(\frac{1}{\left|\mathcal{V}_{n}(e, V)\right|}\right) \mathrm{d} v_{e} \\
& =\frac{1}{\left|\mathcal{V}_{n}(e, V)\right|} \log \left|\mathcal{V}_{n}(e, V)\right| \int_{\mathcal{V}_{n}(e, V)} \mathrm{d} v_{e} \\
& =\log \left|\mathcal{V}_{n}(e, V)\right|,
\end{aligned}
$$

which completes the proof.

We show that entropy production is maximized by the physical measure defined on a cylinder subset of the configuration space restricted to the energy surface.

Proposition 4 The entropy production on the cylinder sets of the configuration space restricted to the energy surface is maximized by the physical measure:

$S\left(\mu_{n, e^{\prime}} \mathcal{V}_{n}(e, V)\right) \leq S\left(\mu_{n, e^{\prime}}^{*} \mathcal{V}_{n}(e, V)\right)$.

Maximization for each value of $t$ maximizes, in turn, the entropy production rate.

Proof We first maximize the entropy production. Starting from Eq. (5), we denote $\mu_{e}^{\prime}=\mathrm{d} \mu_{e} / \mathrm{d} v_{e^{\prime}}$ and use the concavity of the logarithm and Jensen's inequality:

$$
\begin{aligned}
S\left(\mu_{n, e}, \mathcal{V}_{n}(e, V)\right) & =\int_{\mathcal{V}_{n}(e, V)} \mu_{n, e}^{\prime} \log \left(\frac{1}{\mu_{n, e}^{\prime}}\right) \mathrm{d} v_{e} \\
& \leq \log \int_{\mathcal{V}_{n}(e, V)} \mu_{n, e}^{\prime}\left(\frac{1}{\mu_{n, e}^{\prime}}\right) \mathrm{d} v_{e} \\
& =\log \left|\mathcal{V}_{n}(e, V)\right| \\
& =S\left(\mu_{n, e^{\prime}}^{*} \mathcal{V}_{n}(e, V)\right),
\end{aligned}
$$

where the last equality is due to Proposition 3. As our analysis proceeds through fixed time cylinder sets, the maximization holds for each fixed value of $t$ and thus applies to the entropy production rate.

We generalize and strengthen the result of Proposition 4 to the full configuration space restricted to the energy surface, $\mathcal{V}(e, V)$.

Proposition 5 The entropy production on the configuration space restricted to the energy surface is maximized by the physical measure:

$S\left(\mu_{e}, \mathcal{V}(e, V)\right) \leq S\left(\mu_{e}^{*}, \mathcal{V}(e, V)\right)$. 
Maximization for each value of t maximizes, in turn, the entropy production rate.

Proof We analyze at each fixed value of $t$. Replacing $\mathcal{V}_{2}$ with $\mathcal{V}_{1} \cup \mathcal{V}_{2}$, etc., we can assume that the $\mathcal{V}_{i}$ 's are a nested sequence of cylinder sets.

We enclose the finite-energy velocity space in a box $V_{v}$. The cylinder set configuration space is an $n$-dimensional space, whose Lebesgue measure is given by

$\left|\mathcal{V}_{n}\right| \leq \frac{\left|V_{v}\right|^{n}}{n !}$

the inequality reflecting the fact that the box $v_{v}$ may be larger than the velocity space it encloses.

The full configuration space $\mathcal{V}$ is given by the union of the cylinder sets, which we show to have an upper bound:

$|\mathcal{V}|=\left|\bigcup_{n=0}^{\infty} \mathcal{V}_{n}\right| \leq \sum_{n}\left|\mathcal{V}_{n}\right| \leq \sum_{n} \frac{\left|V_{v}\right|^{n}}{n !}=e^{\left|V_{v}\right|}$

We take the logarithm of both sides of (10) (recognizing that, by Proposition 3, the left-hand side is the physical measure entropy of the full configuration space) and obtain the upper bound for $S^{*, \infty}=S\left(\mu_{e}^{*}, \mathcal{V}\right)$ :

$S\left(\mu_{e}^{*}, \mathcal{V}\right)=\log |\mathcal{V}| \leq\left|V_{v}\right|$.

The entropy $S\left(\mathcal{V}_{i}\right)=S\left(\mu_{e}, \mathcal{V}_{i}\right)$ is a monotone increasing functional by formula (6). With $S\left(\mathcal{V}_{i}\right) \nearrow S^{\infty}, S^{*}\left(\mathcal{V}_{i}\right) \nearrow S^{*, \infty}$, and by Proposition $4, S\left(\mathcal{V}_{i}\right) \leq S^{*}\left(\mathcal{V}_{i}\right)$, we conclude that $S^{\infty} \leq S^{*, \infty}$.

\section{Conclusions}

In numerical practice, the subgrid models of the Reynolds stress select a solution of the Navier-Stokes equations. Nonuniqueness of these solutions is known [5], indicating their presumed nonphysical character. The maximum entropy rate production principle can be seen as a necessary admissibility condition to ensure that the solution attained is physically correct.

The dynamic subgrid scale (SGS) models of [18] are a step in this direction, and it can be shown that these dynamic SGS models achieve numerically the physically correct solution for the prediction of solution properties that are isotropic in nature. While dynamic SGS models have a wide acceptance in engineering communities, the usage is not universal.

Published studies $[7,26]$ use no subgrid model at all, and model the Reynolds stress as zero. These solutions are not physically correct, even for isotropic properties of the solution, so that the physical reality of their solutions is suspect. In fact, they do not agree with experimental studies of Rayleigh-Taylor turbulent mixing. The discrepancy is attributed to noise in the experiment. However, the requisite noise was observed to be missing in these experiments [19].

These issues are relevant to the design of an Inertial Confinement Fusion (ICF) capsule, in that larger than predicted instabilities at the ablation surface can lead to early breakthrough of the ablation layer during implosion and to undesired injection of ablation material into the ICF hot spot.

We have proved mathematically that maximum entropy production is a necessary condition to select the physical solution of the Navier-Stokes and Euler equations for incompressible fluids. Experimental validation of the laws of turbulence has focused on other issues, such as the detailed description of multifractal theories. A refining of this body of work might suffice to confirm this dynamic extension of the second law. Future mathematical analysis is also required to extend the result, e.g., to fluid mixing through the transport equation or more general physics.

\section{Compliance with ethical standards}

Conflicts of interest The authors declare that they have no conflict of interest.

Availability of results The mathematical methods and results developed in this paper are fully available to interested researchers.

\section{References}

1. Baron JG, Jauch JM (1972) Entropy, information and Szilard's paradox. Helv Phys Acta 45:220-232

2. Boucher C, Ellis RS, Turkington B (2000) Derivation of maximum entropy principles in two-dimensional turbulence via large deviations. J Stat Phys 98:1235-1278

3. Bourles H (2017) Categories and functors. In: Bourlès H (ed) Fundamentals of advanced mathematics 1: categories, algebraic structures, linear and homological algebra. ISTE Press, Elsevier, pp 1-31

4. Brown D, Cortez R, Minion M (2001) Accurate projection methods for the incompressible Navier Stokes equations. J Comput Phys 168:464-499

5. Buckmaster T, Vicol V (2019) Nonuniqueness of weak solutions to the Navier-Stokes equation. Ann Math 189(1):101-144

6. Buckmaster T, Vicol V (2020) Convex integration and phenomenologies in turbulence. EMS Surv Math Sci 6(1):173-263

7. Cabot W, Cook A (2006) Reynolds number effects on RayleighTaylor instability with possible implications for type la supernovae. Nat Phys 2:562-568

8. Chavanis P, Sommeria J, Robert R (1996) Statistical mechanics of two-dimensional vortices and collisionless stellar systems. Astrophys J 471:385-399 
9. Chefranov SG, Chefranov AS (2019) Exact solution of the compressible Euler-Helmholtz equation and the millennium prize problem generalization. Phys Scr 94(5):054001

10. Chefranov SG, Chefranov AS (2020) The new exact solution of the compressible 3D Navier-Stokes equations. Commun Nonlinear Sci 83:105118

11. Cover T, Thomas J (1991) Elements of information theory. John Wiley, New York

12. Dafermos CM (1973) The entropy rate admissibility criterion for solutions of hyperbolic conservation laws. J Diff Equ 14:202-212

13. Dafermos CM (1989) Regularity and large time behavior of solutions of a conservation law without convexity. Arch Ration Mech Anal 106:243-260

14. Dobovisek A, Vitas M, Brumen M, Fajmut A (2017) Energy conservation and maximal entropy production in enzyme reactions. Biosystems 158:47-56

15. Dunkel J, Talkner P, Hanggl P (2007) Relative entropy, haar measures and relativistic canonical velocity distributions. New J Phys 9(144):1-14

16. Falkovich G, Gawedzski K, Vergassola M (2001) Particles and fields in fluid turbulence. Rev Mod Phys 73:913

17. Gavrilov M, Chétrite R, Bechhoefer J (2017) Direct measurement of weakly nonequilibrium system entropy is consistent with Gibbs-Shannon form. PNAS 114(42):11097-11102

18. Germano M, Piornelli U, Moin P, Cabot W (1991) A dynamic subgrid-scale eddy viscosity model. Phys Fluids 3:1760-1765

19. Glimm J, Cheng B, Sharp DH, Kaman T (2020) A crisis for the verification and validation of turbulence simulations. Phys $D$ 404:132346

20. Glimm J, Sharp DH, Lim H, Kaufman R, Hu W (2015) Euler equation existence, non-uniqueness and mesh converged statistics. Phil Trans R Soc A 373:20140282. https://doi.org/10.1098/ rsta.2014.0282 Los Alamos National Laboratory Preprint LA-UR-14-29521 and Stony Brook University Preprint Number SUNYSB-AMS-15-05

21. del Jesus M, Foti R, Rinaldo A, Rodriguez-Iturbe I (2012) Maximum entropy production, carbon assimilation, and the spatial organization of vegetation in river basins. PNAS 109(51):20837-20841

22. Jordan R, Kinderlehrer D, Otto F (1997) Free energy and the Fokker-Planck equation. Phys D 107:265-271

23. Jordan R, Kinderlehrer D, Otto F (1998) Variational formulation of the Fokker-Planck equation. SIAM J Math Anal 29(1):1-17

24. Lanford O (1973) Entropy and equilibrium states in classical statistical mechanics. Lecture Notes in Physics 20:
25. Leliss CD, Szekelyhidi L (2009) The Euler equations as a differential inclusion. Ann Math 170:1436-1471

26. Livescu D, Wei T, Petersen MR (2011) Direct numerical simulations of Rayleigh-Taylor instability. J Phys Conf Ser 318:082007

27. Lucia U, Grazzini G (2015) The second law today: using maximum-minimum entropy generation. Entropy 17:7786-7797

28. Martyushev LM, Seleznev VD (2006) Maximum entropy production principle in physics, chemistry and biology. Phys Rep 426:1-45

29. Mihelich $M$, Faranda D, Pailard D, Dubrulle $B$ (2017) Is turbulence a state of maximum energy dissipation. Entropy 19:154

30. Noether E (1918) Invariante variationsprobleme. Nachr. d. Konig. Geslich. d. Wiss. zu Gottingen Math-phys. Klasse pp. 183-207

31. Ozawa H, Ohmura A, Lorentz R, Pujol T (2003) The second law of thermodynamics and the global climate system: a review of the maximum entropy production principle. Rev Geophys 41(4):1018

32. Ozawa H, Shimokawa S, Sakuma H (2001) Thermodynamics of fluid turbulence: a unified approach to the maximum transport properties. Phys Rev E 64:026303

33. Prigogine I (1978) Time, structure, and fluctuations. Science 201:777

34. Rao MM (1971) Projective limits of probability spaces. J Multivar Anal 1:28-57

35. Scheffer V (1993) An inviscid flow with compact support in space-time. J Geom Anal 3:343-401

36. Skagerstam BSK (1975) On the notions of entropy and information. J Stat Phys 12(6):449-462

37. Thalabard S, Dubrulle B, Bouchet F (2018) Statistical mechanics of the $3 \mathrm{~d}$ axi-symmetric euler equations in a taylor-couette geometry. arXiv:1306.1081v3

38. Wherl A (1991) The many facets of entropy. Rep Math Phys 30(1):119-129

39. Ziegler H (1963) In: Sneddon IN, Hill R (eds) Progress in Solid Mechanics. North-Holland Publishing, Amsterdam, p 93

40. Ziegler H, Wehrli C (1987) The derivation of constitutive relations from the free energy and the dissipation function. Adv Appl Mech 25:183-238

41. Županović P, Juretić D (2004) Kirchoff's loop law and the maximum entropy production principle. Phys Rev E 70:056108

Publisher's Note Springer Nature remains neutral with regard to jurisdictional claims in published maps and institutional affiliations. 